TITLE:

\title{
Charge-transfer-to-solvent reactions from l- to water, methanol, and ethanol studied by time-resolved photoelectron spectroscopy of liquids
}

\section{$\operatorname{AUTHOR}(S):$}

Okuyama, Haruki; Suzuki, Yoshi Ichi; Karashima, Shutaro; Suzuki, Toshinori

\section{CITATION:}

Okuyama, Haruki ... [et al]. Charge-transfer-to-solvent reactions from I- to water, methanol, and ethanol studied by time-resolved photoelectron spectroscopy of liquids. Journal of Chemical Physics 2016, 145: 074502.

\section{ISSUE DATE:}

2016-08-21

URL:

http://hdl.handle.net/2433/230213

\section{RIGHT:}

This article may be downloaded for personal use only. Any other use requires prior permission of the author and AIP Publishing. The following article appeared in 'The Journal of Chemical Physics 145, 044306 (2016)' and may be found at

https://aip.scitation.org/doi/10.1063/1.4960385.; The full-text file will be made open to the public on 21 August 2017 in accordance with publisher's 'Terms and Conditions for Self-Archiving' 


\section{Charge-transfer-to-solvent reactions from $\mathrm{I}^{-}$to water, methanol, and ethanol studied by time-resolved photoelectron spectroscopy of liquids}

Haruki Okuyama, Yoshi-Ichi Suzuki, Shutaro Karashima, and Toshinori Suzuki

Citation: The Journal of Chemical Physics 145, 074502 (2016); doi: 10.1063/1.4960385

View online: https://doi.org/10.1063/1.4960385

View Table of Contents: http://aip.scitation.org/toc/jcp/145/7

Published by the American Institute of Physics

\section{Articles you may be interested in}

Angle-resolved photoemission spectroscopy of liquid water at $29.5 \mathrm{eV}$

Structural Dynamics 4, 044014 (2017); 10.1063/1.4979857

Photoelectron spectroscopy of aqueous solutions: Streaming potentials of $\mathrm{NaX}(\mathrm{X}=\mathrm{Cl}, \mathrm{Br}$, and $\mathrm{I})$ solutions and electron binding energies of liquid water and $\mathrm{X}^{-}$

The Journal of Chemical Physics 140, 174506 (2014); 10.1063/1.4871877

Full observation of ultrafast cascaded radiationless transitions from $S_{2}(\pi \pi$ ) state of pyrazine using vacuum ultraviolet photoelectron imaging

The Journal of Chemical Physics 145, 044306 (2016); 10.1063/1.4955296

Ultrafast photodynamics of pyrazine in the vacuum ultraviolet region studied by time-resolved photoelectron imaging using 7.8-eV pulses

The Journal of Chemical Physics 145, 044307 (2016); 10.1063/1.4955298

Photoelectron spectroscopy of liquid water, some alcohols, and pure nonane in free micro jets

The Journal of Chemical Physics 106, 9013 (1997); 10.1063/1.474034

A simple electron time-of-flight spectrometer for ultrafast vacuum ultraviolet photoelectron spectroscopy of liquid solutions

Review of Scientific Instruments 85, 103117 (2014); 10.1063/1.4899062

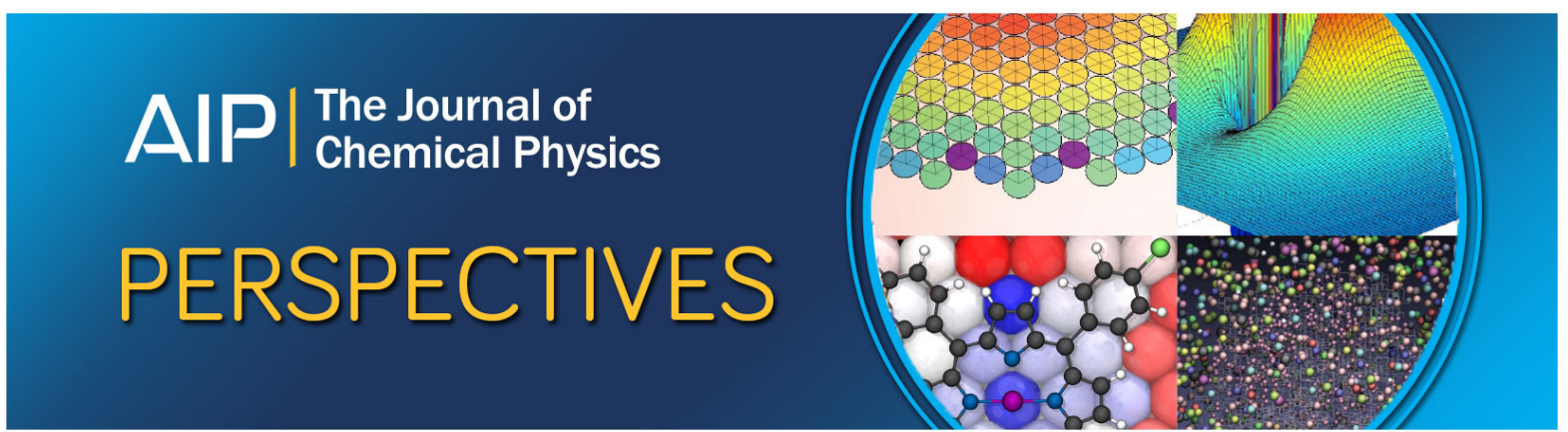




\title{
Charge-transfer-to-solvent reactions from I- to water, methanol, and ethanol studied by time-resolved photoelectron spectroscopy of liquids
}

\author{
Haruki Okuyama, ${ }^{1}$ Yoshi-Ichi Suzuki, ${ }^{1,2}$ Shutaro Karashima, ${ }^{1}$ and Toshinori Suzuki ${ }^{1, a)}$ \\ ${ }^{1}$ Department of Chemistry, Graduate School of Science, Kyoto University, Kitashirakawa-Oiwakecho, \\ Sakyo-ku, Kyoto 606-8502, Japan \\ ${ }^{2}$ Faculty of Pharmaceutical Science, Health Sciences University of Hokkaido, 1757 Kanazawa, Tobetsucho, \\ Ishikari, Hokkaido 061-0293, Japan
}

(Received 28 April 2016; accepted 22 July 2016; published online 15 August 2016)

\begin{abstract}
The charge-transfer-to-solvent (CTTS) reactions from iodide $\left(\mathrm{I}^{-}\right)$to $\mathrm{H}_{2} \mathrm{O}, \mathrm{D}_{2} \mathrm{O}$, methanol, and ethanol were studied by time-resolved photoelectron spectroscopy of liquid microjets using a magnetic bottle time-of-flight spectrometer with variable pass energy. Photoexcited iodide dissociates into a weak complex (a contact pair) of a solvated electron and an iodine atom in similar reaction times, 0.3 ps in $\mathrm{H}_{2} \mathrm{O}$ and $\mathrm{D}_{2} \mathrm{O}$ and $0.5 \mathrm{ps}$ in methanol and ethanol, which are much shorter than their dielectric relaxation times. The results indicate that solvated electrons are formed with minimal solvent reorganization in the long-range solvent polarization field created for $\mathrm{I}^{-}$. The photoelectron spectra for CTTS in $\mathrm{H}_{2} \mathrm{O}$ and $\mathrm{D}_{2} \mathrm{O}$-measured with higher accuracy than in our previous study [Y. I. Suzuki et al., Chem. Sci. 2, 1094 (2011)]—indicate that internal conversion yields from the photoexcited $\mathrm{I}^{-*}$ (CTTS) state are less than $10 \%$, while alcohols provide 2-3 times greater yields of internal conversion from $\mathrm{I}^{-*}$. The overall geminate recombination yields are found to be in the order of $\mathrm{H}_{2} \mathrm{O}>\mathrm{D}_{2} \mathrm{O}>$ methanol $>$ ethanol, which is opposite to the order of the mutual diffusion rates of an iodine atom and a solvated electron. This result is consistent with the transition state theory for an adiabatic outer-sphere electron transfer process, which predicts that the recombination reaction rate has a pre-exponential factor inversely proportional to a longitudinal solvent relaxation time. Published by AIP Publishing. [http://dx.doi.org/10.1063/1.4960385]
\end{abstract}

\section{INTRODUCTION}

Charge-transfer-to-solvent (CTTS) reactions, ${ }^{1-3}$ which involve the transfer of an electron from a solute to its solvent, offer an excellent opportunity to study solvation dynamics in chemical reactions. CTTS reactions of atomic halogen anions are of particular interest, because these solutes have no rovibrational degrees of freedom that would otherwise complicate the dynamics. Among them, CTTS from iodide $\left(\mathrm{I}^{-}\right)$to water has been the most extensively studied, ${ }^{4-22}$ as water is the most important solvent and the UV absorption band of hydrated iodide, $\mathrm{I}^{-}(\mathrm{aq})$, is the most easily accessible; the longest-wavelength $\mathrm{UV}$ absorption band of $\mathrm{I}^{-}(\mathrm{aq})$ is peaked at $225 \mathrm{~nm}$ (220 and $219 \mathrm{~nm}$ in methanol and ethanol, respectively). Although $\mathrm{I}^{-}$in the gas phase has no stable excited state, the characteristic UV absorption bands of $\mathrm{I}^{-}(\mathrm{aq})$ arise from metastable excited states created by solvation. We denote these metastable states as $\mathrm{I}^{-*}$ in this paper (they are often referred to as CTTS states in the literature). Each $\mathrm{I}^{-*}$ state has a singly occupied diffuse molecular orbital, composed primarily of the $6 \mathrm{~s}$ orbital of $\mathrm{I}^{-}$and the neutral iodine core in one of the $\mathrm{I}\left({ }^{2} \mathrm{P}_{3 / 2}\right)$ or $\mathrm{I}\left({ }^{2} \mathrm{P}_{1 / 2}\right)$ spin-orbit states. In the present study, we focus on the $\mathrm{I}^{-*}\left({ }^{2} \mathrm{P}_{3 / 2}\right)$ state and hereafter omit the ${ }^{2} \mathrm{P}_{3 / 2}$ notation.

If we approximate liquid water as a wide gap semiconductor, $\mathrm{I}^{-}(\mathrm{aq})$ is an impurity and its $\mathrm{UV}$ absorption

\footnotetext{
a)E-mail: suzuki@kuchem.kyoto-u.ac.jp
}

occurs within the band gap. For this system, CTTS is the adiabatic separation of an excess electron and an iodine atom to form their weak complex. The complex varies in nature due to the inhomogeneity in the initial solvation structure of $\mathrm{I}^{-}$; however, previous studies have illustrated an average picture of the complex, evolving from a contact pair (CP), which is an excess electron and iodine pair held in the same solvation shell with excess internal energy, to a solvent-separated state (SS), which is a weak complex between a solvated electron and an iodine atom at an equilibrium temperature. ${ }^{13,20}$ The $\mathrm{CP}$ may not be a stable species, ${ }^{6,7}$ but it is considered to be a transient non-stationary state with a lifetime of less than $0.5 \mathrm{ps}$. Some studies have indicated that a neutral halogen atom forms a 1:1 complex with a water molecule. For instance, Sevilla et al. have explained their electron spin resonance (ESR) spectrum of $\mathrm{Cl}$ in water by assuming a $\mathrm{H}_{2} \mathrm{O}-\mathrm{Cl}$ complex; ${ }^{23}$ their calculations at the UMP2/6-31G* level suggested that the $\mathrm{O}-\mathrm{Cl}$ distance is $0.263 \mathrm{~nm}$. A similar 1:1 pair has also been predicted for $\mathrm{Br}^{16}$ and $\mathrm{I}^{17}$ The predicted binding energy between iodine and water is $50 \mathrm{meV}$, and MD simulations suggest that the complex dissociates on a sub-picosecond time scale once a stronger hydrogen-bonding network is constructed around the iodine. ${ }^{17} \mathrm{The}_{2} \mathrm{O}-\mathrm{X}$ complex, if any, is formed after electron photodetachment from $\mathrm{I}^{-}$, and the role of this complex in CTTS remains unclear. Thus, we will not take it into account in this study.

After $\mathrm{I}^{-*}$ is adiabatically separated into an excess electron and an iodine atom, they undergo thermal diffusion that 
competes with geminate recombination in the potential of mean force. These dynamics, which take place in tens of picoseconds or longer, play a decisive role in determining the overall yield of free solvated electron $\left[\mathrm{e}^{-}(\mathrm{solv})\right]$. Staib and Borgis ${ }^{6,7}$ have indicated that the potential of mean force in the CTTS from $\mathrm{Cl}^{-}$to water has a free energy well of $3 k_{\mathrm{B}} T$, where $k_{\mathrm{B}}$ is the Boltzmann constant and $T$ is the temperature. The minimum of this potential was predicted to occur at an electron-chlorine distance of $0.6 \mathrm{~nm}$, which corresponds to a structure with one water molecule between the center of an excess electron density distribution and a chlorine atom. ${ }^{6,7}$ It is noted that since the hydrated electron has a delocalized electron density distribution over multiple hydration shells, ${ }^{24-26}$ one water molecule will not completely attenuate the exchange interactions between the excess electron and the chlorine atom. The potential of mean force becomes flat at a distance of $0.8 \mathrm{~nm}$, which is sufficient for three water molecules to fit in between the excess electron and the chlorine atom. Based on the theoretical calculations of Staib and Borgis, Kloepfer et al. have analyzed their transient absorption spectra of CTTS from $\mathrm{I}^{-}$to water $^{10}$ and suggested that the potential minimum lies at $0.4 \mathrm{~nm}$ and has a well depth of $620 \mathrm{~cm}^{-1} \cdot{ }^{10} \mathrm{In}$ a similar study carried out with higher time resolution over a wider probe wavelength region, Iglev et al. proposed that the potential minimum occurs at $0.6 \mathrm{~nm}$ and that the depth is $850 \pm 100 \mathrm{~cm}^{-1} .{ }^{14}$

Previously, we reported the first time-resolved photoelectron spectroscopy (TRPES) ${ }^{18}$ study of the CTTS reaction from $\mathrm{I}^{-}$to bulk water using liquid microjets. ${ }^{20}$ TRPES using sub-picosecond UV pulses has revealed a rapid increase in the electron binding energy (eBE) over time, corresponding to evolution from $\mathrm{CP}$ to a hydrated electron $\left[\mathrm{e}^{-}(\mathrm{aq})\right]$ via $\mathrm{SS}^{20}$ However, the low electron detection efficiency of hemispherical electron energy analyzers has limited the signal-to-noise ratio and accuracy in measurements of low-energy electrons $(<3 \mathrm{eV})$. Thus, our recent TRPES studies $^{27,28}$ on other reactions were performed using a magnetic bottle time-of-flight (MBTOF) photoelectron spectrometer ${ }^{28}$ with variable pass energy, which provides several orders higher signal count rate and improved accuracy in measurements of low-energy electrons compared to a hemispherical analyzer. Thus, the present study revisits the CTTS reaction from $\mathrm{I}^{-}$to bulk water with the superior MBTOF apparatus. The new experimental data enable refinement of our previous study on the isotope effect in CTTS reactions in water. ${ }^{20}$ Moreover, we investigate similar CTTS dynamics from $\mathrm{I}^{-}$to methanol and ethanol to gain further insight into the CTTS dynamics in polar protic solvents. Previously, Vilchiz et al. ${ }^{12}$ studied CTTS from $\mathrm{I}^{-}$ to alcohols using transient absorption spectroscopy (TAS), but their study focused only on the long-time dynamics of diffusion and recombination. The present study considers the dynamics more thoroughly, beginning from ultrafast solvation and including the long-time diffusion and recombination processes. We employ both kinetic and diffusion models for the data analysis, as well as compare our results on methanol with those of a recent TRPES study by Elkins et $_{\text {al. }}{ }^{22}$

\section{EXPERIMENTAL SECTION}

\section{A. Liquid microjet}

We employed $\mathrm{NaI}$ as a solute and $\mathrm{H}_{2} \mathrm{O}, \mathrm{D}_{2} \mathrm{O}$, methanol, and ethanol as the solvents. The concentration of the solutions was adjusted to $40-100 \mathrm{mM}$ to minimize electrokinetic charging, which occurs for liquids discharged from a fused silica capillary nozzle. The sample solution was pressurized using a high-performance liquid chromatography (HPLC) gradient flow pump and degassed using an in-line degasser prior to discharging from the capillary $(15 \mu \mathrm{m}$ i.d., $10 \mathrm{~mm}$ length). The outer surface of the capillary was coated with fine graphite to prevent it from charging up. The laser beams were crossed with the liquid microjet at around $1 \mathrm{~mm}$ downstream from the nozzle. The sample solutions and the nozzle assembly were kept at room temperature, while the liquid microjet temperature was low, owing to evaporative cooling of the jet in vacuum. When the jet diameter is small $(<10 \mu \mathrm{m})$, evaporated gas effuses into the vacuum at the liquid temperature, while the liquid microjet is cooled, as predicted by the Clausius-Clapeyron equation. ${ }^{29,30}$ When the microjet diameter is larger, evaporated gas stagnates above the liquid surface, and its free expansion lowers the gas temperature to below that of the liquid surface. The liquid is less efficiently cooled than expected from the Clausius-Clapeyron equation because a fraction of the stagnated gas recondenses into the liquid. This behavior has been confirmed experimentally for liquid microjets. The temperatures of our liquid microjets were estimated to be around $278 \mathrm{~K}$ for water and $260 \mathrm{~K}$ for alcohol based on the Raman thermometry study by Wilson et al. ${ }^{31}$ At these temperatures, the mutual diffusion coefficients of a pair of iodine and $\mathrm{e}^{-}$(solv) in $\mathrm{H}_{2} \mathrm{O}, \mathrm{D}_{2} \mathrm{O}$, methanol, and ethanol are estimated to be $3.0,2.6,1.6$, and $0.8 \times 10^{-5} \mathrm{~cm}^{2} / \mathrm{s}$, as explained in the supplementary material. ${ }^{47}$ We have corrected the observed photoelectron kinetic energies (PKEs) for streaming potentials using the method described by Kurahashi et al. ${ }^{32}$

\section{B. Laser system}

The experiments were performed using a $1-\mathrm{kHz}$ Ti:sapphire regenerative amplifier (Quantronix Titan; $100 \mathrm{fs,}$ $2 \mathrm{~mJ} /$ pulse, $800 \mathrm{~nm}$ ) and two collinear optical parametric amplifiers (Light Conversion TOPAS). The output of one of the optical parametric amplifiers was mixed with $800 \mathrm{~nm}$ to generate the pump pulses at $225 \mathrm{~nm}$, while the $260-\mathrm{nm}$ output from the other was used as the probe pulses. The probe pulses were optically time-delayed with respect to the pump pulses using a corner cube mounted on a computercontrolled linear translation stage. Using concave mirrors, the pump and probe pulses were gently focused on the liquid microjet, at which the laser beam diameters were estimated to be $100 \mu \mathrm{m}$. The typical pump and probe pulse energies were $1.5-4.0 \mathrm{~nJ} /$ pulse and $3.6-13.0 \mathrm{~nJ} /$ pulse, respectively, and the cross-correlation time between them was measured using $\left(1+1^{\prime}\right)$ two-color resonance enhanced multiphoton ionization of NO to be $250-300$ fs. Based on the density of iodide $\left(6.4 \times 10^{-2} \mathrm{~nm}^{-3}\right)$ and photoexcitation efficiency 
$(0.1 \%)$, the density of photoexcited iodide is estimated to be $6.4 \times 10^{-5} \mathrm{~nm}^{-3}$.

\section{TRPES of liquids}

Photoemission from liquids is understood using a threestep model, which assumes (1) promotion of an electron into the conduction band of solvent, (2) transport of the electron to the liquid-gas interface, and (3) ejection of the electron from the interface. The upper states reached in step (1) can be either a delocalized electronic state and/or some metastable resonance states with unknown character. In step (2), an electron wave packet travels through the solvent, in which elastic and inelastic scattering can occur with solvent and/or solute molecules. ${ }^{27,28,33,34}$

Since the PKE created in our UV photoemission experiment is smaller than the electronic excitation energy of the solvents $(\sim 7 \mathrm{eV})$, inelastic scattering in solution is primarily vibrational. ${ }^{35}$ Although the cross section of vibrational inelastic scattering is smaller than that of electronic inelastic scattering, its finite magnitude determines the effective attenuation length of low-energy electrons in solvent. Since elastic scattering deflects the velocity directions of electrons and increases the effective path length in liquid, it increases the probabilities of inelastic scattering events. Although effective attenuation lengths at low PKEs $(<10 \mathrm{eV})$ have not been established, experimental studies suggest that they are on the order of nanometers..$^{33,36-40}$

\section{Magnetic bottle spectrometer}

The photoionization chamber houses a liquid beam nozzle on a 3-axis manipulator and a SmCo magnet with a conical iron cap on another 3-axis manipulator. The tip of the magnet was placed $2 \mathrm{~mm}$ from the photoionization point. The photoionization chamber was evacuated with a $1600 \mathrm{l} / \mathrm{s}$ turbo molecular pump and a liquid nitrogen trap employed for cryo-pumping. A typical pressure in the chamber was $10^{-4}$ Torr when running a liquid jet. The liquid jet traveled vertically downward in the photoionization chamber and was trapped in a vessel cooled with liquid nitrogen. An entrance skimmer $(\varnothing 0.5 \mathrm{~mm})$ for an electron TOF analyzer was located $2 \mathrm{~mm}$ from the ionization point. The analyzer, which contains a solenoid coil and a $\varnothing 40 \mathrm{~mm}$ microchannel plate (MCP) detector, was evacuated using a 2600 1/s turbo molecular pump. The electric potential of the MCP surface was kept at $+300 \mathrm{~V}$ to ensure that the detection sensitivity was independent of the initial PKE. A grounded wire mesh was placed in front of the MCP to prevent leakage of the electric field due to the positive voltage into the field-free region. The signal from the MCP was amplified using a preamplifier (FAST ComTec, TA2000B-3) and counted with a multichannel scaler (FAST ComTec, P7887). The electron flight path from the ionization point to the detector was $1090 \mathrm{~mm}$. The magnetic flux densities at the ionization point and in the flight tube were estimated to be $0.4 \mathrm{~T}$ and $1 \mathrm{mT}$, respectively. Both the photoionization chamber and TOF analyzer were shielded against external magnetic fields by a permalloy inner layer.
The electron detection efficiency of a standard MBTOF spectrometer diminishes for low PKEs of $<0.5 \mathrm{eV}$. In this study, we have employed an MBTOF spectrometer with a variable electric potential in the electron flight region to increase the kinetic energies of photoelectrons in the analyzer. Prior to the measurements, we have confirmed that the electron transmission curve of our MBTOF spectrometer was flat for PKEs higher than $0.3 \mathrm{eV}$. Therefore, we applied a voltage of $+0.5 \mathrm{~V}$ to the analyzer to make the electron pass energy through the analyzer higher than $0.5 \mathrm{eV}$, which ensured that the detection efficiency was uniform over the entire PKE region. For a $0.1 \mathrm{M}$ aqueous $\mathrm{NaI}$ solution, an average number of $\sim 2.5$ electrons were detected per laser shot at pump and probe pulse energies of 3 and $13 \mathrm{~nJ} /$ pulse, respectively.

\section{MODELS FOR DATA ANALYSIS}

In this study, we employed both kinetic and diffusion models for analyzing our TRPES data and examined the consistency between them. Although the kinetic model is easily implemented, it does not describe the long-time diffusion rigorously. The diffusion model enables systematic interpretation of the observed time profiles through use of the diffusion constants. In this section, the two models are briefly explained.

\section{A. Kinetic model}

We have performed a global fit of the time-dependent electron binding energy distribution (eBED) by assuming that each transient species has a time-independent photoelectron spectrum and a time-dependent population. As we reported previously, ${ }^{20}$ the observed photoelectron intensity profile exhibits at least three exponentially decaying components. Thus, we assumed two reaction intermediates, CP and SS, in our kinetic model, as shown in Fig. 1.

With this kinetic model, the relative population in each state after photoexcitation to $\mathrm{I}^{-*}$ is expressed as follows:

$$
\left[\mathrm{I}^{-*}\right]=e^{-t / \tau_{a}},
$$

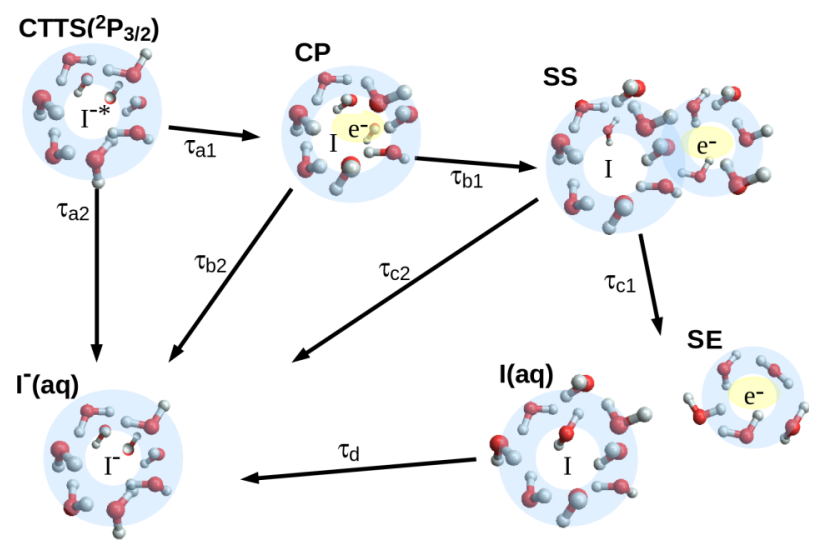

FIG. 1. Graphical representation of our kinetic model for the CTTS reaction from $\mathrm{I}^{-}$to bulk water. $\tau_{d}$ in the model corresponds to the overall depopulation time of the solvated electron (SE), during which geminate recombination is dominant. 


$$
\begin{gathered}
{[\mathrm{CP}]=r_{a} \tau_{b}\left(\frac{e^{-t / \tau_{a}}}{\tau_{a b}}+\frac{e^{-t / \tau_{b}}}{\tau_{b a}}\right),} \\
{[\mathrm{SS}]=r_{a} r_{b} \tau_{c}\left(\frac{\tau_{a} e^{-t / \tau_{a}}}{\tau_{a b} \tau_{a c}}+\frac{\tau_{b} e^{-t / \tau_{b}}}{\tau_{b a} \tau_{b c}}+\frac{\tau_{c} e^{-t / \tau_{c}}}{\tau_{c a} \tau_{c b}}\right),} \\
{\left[\mathrm{e}^{-}(\mathrm{solv})\right]=r_{a} r_{b} r_{c} \tau_{d}\left(\frac{\tau_{a}^{2} e^{-t / \tau_{a}}}{\tau_{a b} \tau_{a c} \tau_{a d}}+\frac{\tau_{b}^{2} e^{-t / \tau_{b}}}{\tau_{b a} \tau_{b c} \tau_{b d}}\right.} \\
\left.+\frac{\tau_{c}^{2} e^{-t / \tau_{c}}}{\tau_{c a} \tau_{c b} \tau_{c d}}+\frac{\tau_{d}^{2} e^{-t / \tau_{d}}}{\tau_{d a} \tau_{d b} \tau_{d c}}\right)
\end{gathered}
$$

where $\tau_{\xi}=\left(\tau_{x 1}^{-1}+\tau_{x 2}^{-1}\right)^{-1}$ and $\tau_{x y}=\tau_{x}-\tau_{y}(x, y=a, b, c$, and $d$ ). The branching ratio in each kinetic step is denoted by $r_{x}\left(=\tau_{x} \tau_{x 1}^{-1}\right)$. The recombination time constants $\tau_{x 2}$ are obtained from the fitting parameters $\tau_{x}$ and $r_{x}$ as $\tau_{x 2}=\tau_{x}\left(1-r_{x}\right)^{-1}$. If all transient species have an identical photodetachment cross section $\sigma_{i},{ }^{20}$ the photoelectron signal intensity is expressed by

$$
\begin{aligned}
I\left(t, E_{k}\right)= & \sigma_{i}\left(c_{a k}\left[\mathrm{I}^{-*}\right]+c_{b k}[\mathrm{CP}]+c_{c k}[\mathrm{SS}]\right. \\
& \left.+c_{d k}\left[e^{-(\mathrm{solv})}\right]\right) \otimes g(t)
\end{aligned}
$$

where $k$ is an index for equally spaced PKE bins (typically $0.2 \mathrm{eV})$. The expansion coefficients $c_{x k}(x=a, b, c, d)$ provide the eBED intensities of each chemical species in the $k$ th energy bin. The function $g(t)$ is a Gaussian cross-correlation function representing our laser pulses. It is possible that the actual photodetachment cross sections of each species differ; however, they are not separated mathematically from the $r_{x}$ values and cannot be rigorously determined. Thus, the validity of the assumed identical cross sections is discussed later in combination with the $r_{x}$ values extracted from the data analysis.

\section{B. Diffusion model}

The photoelectron signal intensity at long times (>several ps) following photoexcitation of $\mathrm{I}^{-}$is primarily determined by mutual diffusion and recombination of a solvated electron and an iodine atom, which can be analyzed using the Smoluchouski diffusion equation. On the other hand, the initial reaction step from $\mathrm{I}^{-*}$ to $\mathrm{CP}$ (or SS) is not a diffusion process, and it should be described as a kinetic step(s). As the minimal assumption necessary for our diffusion model, we employed only an exponential decay of $\mathrm{I}^{-*}$ with time constant $\tau_{a}$ to form a weak complex; the complex is generated at an electron-iodine distance of $r_{0}$ in the potential of mean force with time-dependence $r_{a}\left\{1-\exp \left(-t / \tau_{a}\right)\right\}$, where $r_{a}$ is the quantum yield of the complex. As we show later, least squares fitting without assuming a kinetic step can also reproduce the observed time profile; however, it requires an unrealistic potential of mean force. In our diffusion model analysis, no photoelectron spectral evolution is taken into account; in fact, the spectral evolution is negligible after formation of a weak complex of an iodine atom and an electron.

The probability density, $c(t, r)$, of a solvated electron around an iodine atom depends on the elapsed time $(t)$ after irradiation by a pump pulse and the iodine-electron distance $(r)$. We assume spherical symmetry for the probability density ${ }^{41}$ and a sufficiently low concentration of $\mathrm{I}^{-*}$ so that only a single electron-iodine pair in the system can be considered. The diffusion equation is written as

$$
\frac{\partial \psi(t, r)}{\partial t}=\hat{L} \psi(t, r)
$$

where $\psi(t, r)$ and $\hat{L}$ are, respectively,

$$
\psi(t, r)=4 \pi r^{2} c(t, r)
$$

and

$$
\hat{L}=D^{\prime} \frac{\partial}{\partial r} r^{2} e^{-V(r)} \frac{\partial}{\partial r} r^{-2} e^{V(r)} .
$$

In the above, $V(r)$ is the potential of mean force (in units of $\left.k_{\mathrm{B}} T\right)$, and $D^{\prime}$ is the mutual diffusion constant.

Equation (6) is solved numerically by discretizing the radial coordinate $r$ with an equal distance $\Delta r$ of $5 \mathrm{pm}$. When expressing $\psi(t, r)$ in each cell as $q_{i}(t)(i=1,2, \ldots, \mathrm{N}), q_{i}(t)$ obeys the following equation:

$$
\begin{aligned}
\frac{d}{d t} q_{i}(t)= & \Omega(i \mid i-1) q_{i-1}(t)-[\Omega(i-1 \mid i)+\Omega(i+1 \mid i)] \\
& \times q_{i}(t)+\Omega(i \mid i+1) q_{i+1}(t),
\end{aligned}
$$

where $\Omega(i \mid j)$ is the transition probability from $j$ to $i$ per unit time. Equation (9) is equivalently expressed in vector-matrix form as

$$
\frac{d}{d t} \mathbf{q}=\mathbf{L} \cdot \mathbf{q}
$$

We assume that geminate recombination occurs at the contact radius $r_{\text {contact }}\left(r_{\text {contact }}<r_{0}\right)$. As a boundary condition, geminate recombination is introduced at $r_{\text {contact }}$ as $\Omega(0 \mid 1)=\kappa_{r} / \Delta r$. Equation (10) is solved by diagonalizing the matrix $\mathbf{L}$, and the solution is obtained in the following form:

$$
q_{i}(t)=\sum_{j=1}^{N} a_{i j} \exp \left(-\frac{t}{\tau_{j}}\right),
$$

where $a_{i j}$ are the constants that depend on the initial conditions, and $-1 / \tau_{j}$ are the eigenvalues of $\mathbf{L}$. Then, the total photoelectron intensity is expressed by

$$
I(t)=\sigma_{i}\left[e^{-\frac{t}{\tau_{a}}}+\sum_{i=1}^{N} q_{i}(t)\right] \otimes g(t),
$$

where $g(t)$ is the Gaussian cross-correlation function between the pump and probe pulses. Here, we have assumed the photodetachment cross section $\sigma_{i}$ to be independent of $r$.

We have approximated the attractive potential of mean force using the Morse function,

$$
V(r)=\Delta G\left(1-e^{-\beta\left(r-r_{\min }\right)}\right)^{2}-\Delta G,
$$

with the potential minimum $r_{\text {min }}$ taken to be the initial distance $r_{0}$. Previously, Kloepfer et al. determined a value of $\kappa_{r}=0.5 \mathrm{~nm} / \mathrm{ps}$, assuming $r_{0}=r_{\text {contact }}=0.4 \mathrm{~nm},{ }^{10}$ while Iglev et al. determined $r_{\text {contact }}=0.5 \pm 0.01 \mathrm{~nm}$, assuming $r_{0}=0.61 \mathrm{~nm}$ and $\kappa_{r}=\infty$ (the absorbing boundary condition). ${ }^{14}$ In our analysis, we also assumed a value of $r_{0}=r_{\text {contact }}=0.4 \mathrm{~nm}$ to determine the five parameters $\tau_{a}$, $r_{a}, \Delta G, \beta$, and $\kappa_{r}$. Iglev et al. ${ }^{14}$ argued that the value of $r_{\text {contact }}=0.4 \mathrm{~nm}$ assumed by Kloepfer et al. ${ }^{10}$ is too small and attributed it to be the source of their shallower interaction 
potential; however, this argument is not quite correct. In fact, a smaller assumed $r_{0}$ and $r_{\text {contact }}$ should provide a deeper interaction potential, so the shallower potential estimated by Kloepfer et al. ${ }^{10}$ should be due to other factors. Since it was difficult for us to accurately determine the absolute values of $r_{0}$ and $r_{\text {contact }}$, we assumed $r_{0}=r_{\text {contact }}=0.4 \mathrm{~nm}$ in all of our analyses; however, this simplification does not qualitatively alter our conclusion.

\section{RESULTS AND ANALYSIS}

\section{A. CTTS in water and kinetic model analysis}

Figure 2 shows (a) the two-dimensional (2D) map of the eBED measured for CTTS in $\mathrm{H}_{2} \mathrm{O}$, (b) the global fit using the kinetic model, and (c) the residue of the fit. The time axis is logarithmic. The global fit well reproduces the experimental 2D map with the best-fit parameters listed in Table I. The overall decay time constants, $\tau_{a}, \tau_{b}$, and $\tau_{c}$, are in agreement with our previous study and also with the time constants of $0.22 \pm 0.05,0.7 \pm 0.05$, and $21 \pm 2$ ps reported by Iglev et al. using TAS for a $2.5 \mathrm{mM} \mathrm{NaI}$ solution at $298 \mathrm{~K}^{.14}$ It is worth noting that depopulation of the $\mathrm{I}^{-*}$ state may not occur immediately after photoexcitation and that the signal exhibits a plateau. Careful analysis of the results shown in Fig. 4 and similar data obtained with a higher time resolution of $125 \mathrm{fs}$ indicates that the experimental results can also be explained by assuming an induction time of about 50 fs prior to $\mathrm{I}^{-*}$ population decay and a decay time constant $\left(\tau_{a}\right)$ of $\sim 100$ fs. This fact seems to accord with the $60 \mathrm{fs}$ component observed by Chergui and colleagues using ultrafast fluorescence spectroscopy of this reaction; ${ }^{21}$ however, the time resolution of the present study is not quite sufficient to elucidate the sub-100 fs dynamics clearly.

Figure 4 shows the decay-associated spectra determined for the four solvents using the global fit. The spectral intensities of $\mathrm{CP}$, SS, and $\mathrm{e}^{-}\left(\right.$solv) in $\mathrm{H}_{2} \mathrm{O}$ and $\mathrm{D}_{2} \mathrm{O}$ appear stronger than those of our previous study due to the improved detection sensitivity for low-energy electrons and

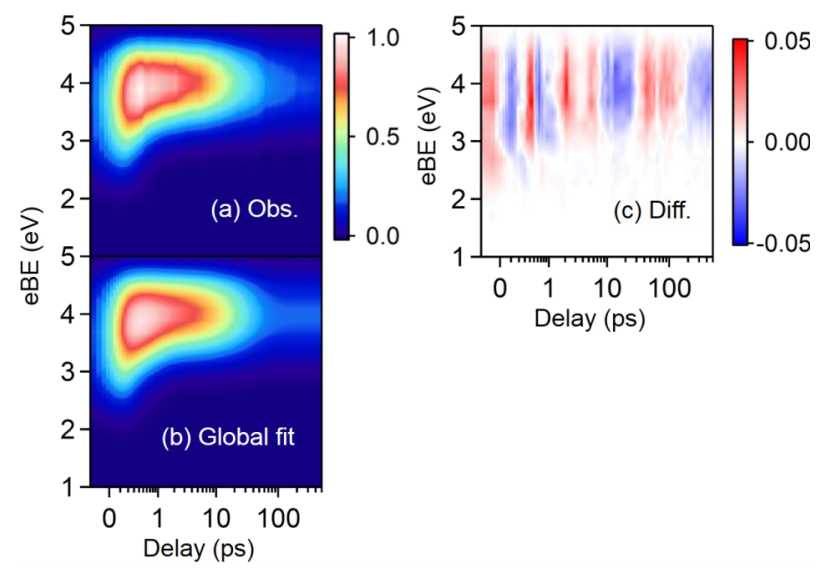

FIG. 2. (a) Time-evolution of the eBED measured for the CTTS reaction from $\mathrm{I}^{-*}$ to $\mathrm{H}_{2} \mathrm{O}$, (b) simulated distribution obtained by least squares fitting based on the kinetic model, and (c) the difference between (a) and (b). The pump and probe wavelengths are 225 and $260 \mathrm{~nm}$, respectively. A constant has been added to the actual delay $\left(t_{\mathrm{plot}}=t_{\text {true }}+0.2 \mathrm{ps}\right)$ to shift the entire distribution and display the data around $t=0$. The time label and grids are presented for $t_{\text {true }}$.

accurate calibration of the electron transmission curve of the electron spectrometer. The integrated area of each spectrum in Fig. 3 provides the quantum yield multiplied by the relative photodetachment cross section of each species. By assuming the photodetachment cross sections of $\mathrm{I}^{-*}$ and $\mathrm{CP}$ to be identical, the quantum yield $\left(\mathrm{r}_{a}\right)$ of $\mathrm{CP}$ is estimated to be 0.95 in $\mathrm{H}_{2} \mathrm{O}$ and 0.89 in $\mathrm{D}_{2} \mathrm{O}$, as listed in Table I. These values are greater than those of 0.60 and 0.54 estimated in our previous study. ${ }^{20}$ This discrepancy is ascribed to the low detection efficiency of low-energy electrons and incomplete calibration of the hemispherical electron analyzer in our previous study.

If the true quantum yield of $\mathrm{CP}$ is unity, the smaller electron signal intensity from $\mathrm{CP}$ implies that the detachment cross section of $\mathrm{CP}$ is lower than that of $\mathrm{I}^{-*}$. In either cases, this study clearly shows that the yield of CP is greater than 0.9 and that quenching (internal conversion) of $\mathrm{I}^{-*}$ is not significant. CTTS in $\mathrm{H}_{2} \mathrm{O}$ and $\mathrm{D}_{2} \mathrm{O}$ exhibits almost indistinguishable eBEDs, as can be seen in Fig. 3, as well as very similar $\mathrm{I}^{-*}$

TABLE I. Kinetic time constants (ps) and branching ratios determined for each sample.

\begin{tabular}{lccccccc}
\hline \hline & $\mathrm{NaI} / \mathrm{H}_{2} \mathrm{O}$ & $\mathrm{NaI} / \mathrm{H}_{2} \mathrm{O}^{\mathrm{a}}$ & $\mathrm{NaI} / \mathrm{D}_{2} \mathrm{O}$ & $\mathrm{NaI} / \mathrm{D}_{2} \mathrm{O}^{\mathrm{a}}$ & $\mathrm{NaI} / \mathrm{MeOH}$ & $\mathrm{NaI} / \mathrm{MeOH}^{\mathrm{b}}$ & $\mathrm{NaI} / \mathrm{EtOH}$ \\
\hline$\tau_{a}$ & $0.23 \pm 0.03$ & $0.23 \pm 0.1$ & $0.25 \pm 0.01$ & $0.31 \pm 0.1$ & $0.45 \pm 0.09$ & $0.55 \pm 0.11$ & $0.44 \pm 0.10$ \\
$\tau_{b}$ & $0.87 \pm 0.29$ & $1.1 \pm 0.2$ & $1.1 \pm 0.2$ & $1.33 \pm 0.2$ & $7.4 \pm 1.3$ & $8 \pm 3$ & $3.7 \pm 1.1$ \\
$\tau_{c}$ & $23 \pm 2.0$ & $21 \pm 2$ & $37 \pm 1.7$ & $37 \pm 4$ & $65 \pm 9.5$ & $41 \pm 7$ & $119 \pm 25$ \\
$\tau_{d}$ & $2.1 \times 10^{9 c}$ & $300-1600$ & $2.6 \times 10^{9 c}$ & $400-1400$ & $1.1 \times 10^{8 c}$ & & $2.6 \times 10^{15 c}$ \\
$\tau_{a 1}$ & $0.25 \pm 0.04$ & $0.4 \pm 0.1$ & $0.29 \pm 0.03$ & $0.6 \pm 0.1$ & $0.64 \pm 0.14$ & 0.55 & $0.55 \pm 0.13$ \\
$\tau_{a 2}$ & $4.1 \pm 5.7$ & $0.6 \pm 0.1$ & $2.1 \pm 1.2$ & $0.7 \pm 0.1$ & $1.6 \pm 0.47$ & & $2.1 \pm 0.57$ \\
$\tau_{b 1}$ & $1.3 \pm 0.4$ & $1.7 \pm 0.3$ & $1.7 \pm 0.3$ & $2.5 \pm 0.3$ & $8.2 \pm 1.4$ & 7.7 & $3.7 \pm 1.1$ \\
$\tau_{b 2}$ & $2.6 \pm 0.9$ & $3.5 \pm 0.5$ & $3.1 \pm 0.6$ & $2.9 \pm 0.4$ & $73 \pm 18.5$ & & $1171 \pm 3240$ \\
$\tau_{c 1}$ & $105 \pm 12$ & $84 \pm 10$ & $135 \pm 11$ & $128 \pm 20$ & $115 \pm 17.5$ & 57 & $162 \pm 37$ \\
$\tau_{c 2}$ & $29 \pm 3$ & $28 \pm 2$ & $50 \pm 3$ & $52 \pm 5$ & $147 \pm 23$ & 146 & $452 \pm 138$ \\
$r_{a}$ & $0.94 \pm 0.08$ & $0.60 \pm 0.05$ & $0.88 \pm 0.07$ & $0.54 \pm 0.04$ & $0.72 \pm 0.06$ & 1 & $0.79 \pm 0.03$ \\
$r_{b}$ & $0.66 \pm 0.02$ & $0.67 \pm 0.01$ & $0.64 \pm 0.03$ & $0.53 \pm 0.01$ & $0.90 \pm 0.02$ & 1 & $1.00 \pm 0.01$ \\
$r_{c}$ & $0.21 \pm 0.02$ & $0.25 \pm 0.01$ & $0.27 \pm 0.02$ & $0.29 \pm 0.02$ & $0.56 \pm 0.02$ & 0.71 & $0.74 \pm 0.06$ \\
\hline \hline
\end{tabular}

\footnotetext{
${ }^{\text {aReference } 20 .}$

${ }^{\mathrm{b}}$ Reference 22.

${ }^{\mathrm{c}}$ These values were too large to determine accurately in our measurements.
} 


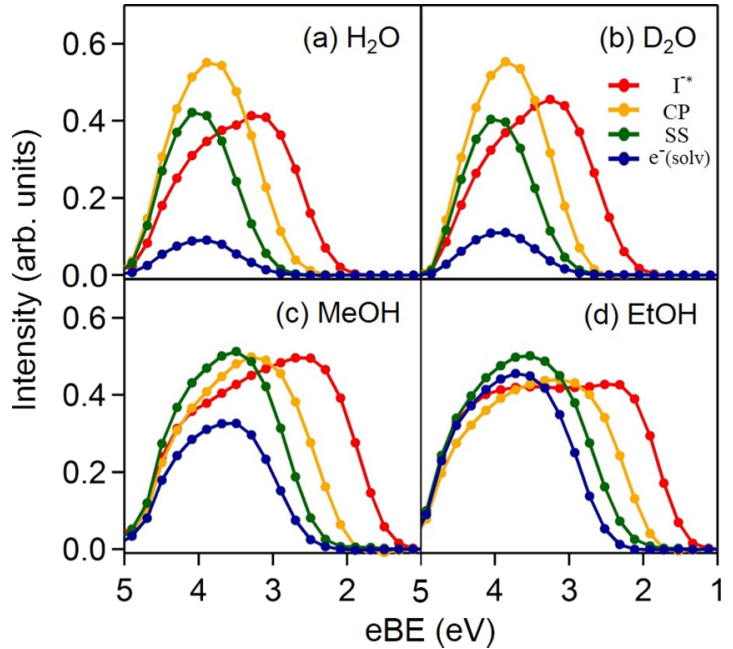

FIG. 3. Decay-associated spectra determined for CTTS reactions from $\mathrm{I}^{-*}$ to (a) $\mathrm{H}_{2} \mathrm{O}$, (b) $\mathrm{D}_{2} \mathrm{O}$, (c) methanol, and (d) ethanol. The red, yellow, green, and blue curves correspond to $\mathrm{I}^{-*}, \mathrm{CP}, \mathrm{SS}$, and $\mathrm{e}^{-}(\mathrm{solv})$. The pump and probe wavelengths are 225 and $260 \mathrm{~nm}$, respectively.

lifetimes of $\tau_{a}=0.25$ ps and 0.24 ps (Table I), respectively. The quantum yields, $r_{b}$ and $r_{c}$, and associated time constants $\tau_{b 1}, \tau_{b 2}, \tau_{c 1}$, and $\tau_{c 2}$ are in excellent agreement with our previous study.

\section{B. CTTS in alcohol and kinetic model}

We performed similar experiments on the CTTS reactions from $\mathrm{I}^{-}$to methanol and ethanol. The time-energy maps of eBED presented in Fig. 4 clearly indicate that alcohol solutions exhibit higher quantum yields of $\mathrm{e}^{-}(\mathrm{solv})$. The global fits (not shown here) using the kinetic model well reproduced the observed eBEDs. The best-fit time constants and branching ratios are tabulated in Table I. Our spectra measured for methanol exhibit greater bandwidth than those reported by Elkins et al., ${ }^{22}$ which suggests that the

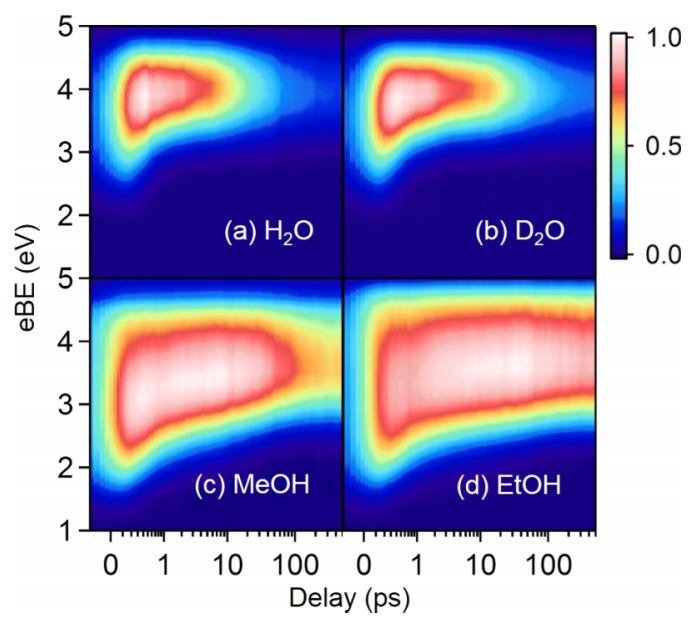

FIG. 4. Time-evolution of the eBED measured for the CTTS reaction from $\mathrm{I}^{-*}$ to (a) $\mathrm{H}_{2} \mathrm{O}$, (b) $\mathrm{D}_{2} \mathrm{O}$, (c) methanol, and (d) ethanol. The pump and probe wavelengths are 225 and $260 \mathrm{~nm}$, respectively. A constant has been added to the actual delay $\left(t_{\text {plot }}=t_{\text {true }}+0.2 \mathrm{ps}\right)$ to shift the entire distribution and display the data around $t=0$. The time label and grids are presented for $t_{\text {true }}$. electron transmission efficiency of their magnetic bottle spectrometer diminishes at low electron kinetic energy. Nonetheless, the extracted time constants are in reasonable agreement between the two studies.

Although the reaction times from $\mathrm{I}^{-*}$ to $\mathrm{CP}$ are slightly longer than that in water, they are still less than 1 ps. Interestingly, the reaction time $\left(\tau_{a 1}\right)$ in ethanol is smaller than that in methanol. The $r_{a}(0.7-0.8)$ values for the alcohols are smaller than that in water, while the overall quantum yields $\left(\phi_{\mathrm{SE}}=r_{a} r_{b} r_{c}\right)$ of $\mathrm{e}^{-}$(solv) are far greater, which is primarily due to the larger $r_{\mathrm{c}}$ values. If we neglect $r_{a}$ and $r_{b}$, the survival probability of $\mathrm{e}^{-}$(solv) is given by $r_{\mathrm{c}}$ to be 0.56 and 0.74 for methanol and ethanol, respectively. These values are smaller than the corresponding values of 0.70 and 0.85 previously reported by Vilchiz et al. using TAS of the solutions at room temperature. ${ }^{12}$ The differences are most likely the result of the lower temperature of our liquid microjet (estimated to be $260 \mathrm{~K})$

The decay-associated spectra extracted for methanol and ethanol are shown in Fig. 3. The photoelectron spectra for the CTTS reactions in alcohol are much broader than that in water. Specifically, the solvated electron bandwidth increases in the order of water $(1.3)<$ methanol $(1.6)<$ ethanol $(1.9 \mathrm{eV})$ at the probe wavelength of $260 \mathrm{~nm}\left(\mathrm{~h} v_{\text {probe }}=4.8 \mathrm{eV}\right) .{ }^{28}$ The best estimates for the vertical electron binding energies (VBEs) of solvated electrons in water, methanol, and ethanol have been reported to be 3.3, 3.1, and 3.1 eV, respectively, ${ }^{18,28,42-46}$ while Fig. 4 indicates higher VBE values. The photoelectron spectra of $\mathrm{I}^{-*}$ are rather broad in all cases, which is due to inelastic scattering in solution, as discussed in detail elsewhere. ${ }^{28}$

\section{Diffusion model analysis}

Figures 5 and 6 show a comparison of the observed photoelectron time profile and the diffusion model simulation for methanol and ethanol, respectively. The best-fit parameters employed are summarized in Table II. We assumed $r_{a}=r_{\min }$ $=0.4 \mathrm{~nm}$ in all cases. The well depths $\Delta G$ of the potential of mean force estimated for methanol and ethanol are smaller than those reported by Vilchiz et al. ${ }^{12}$ The discrepancy is

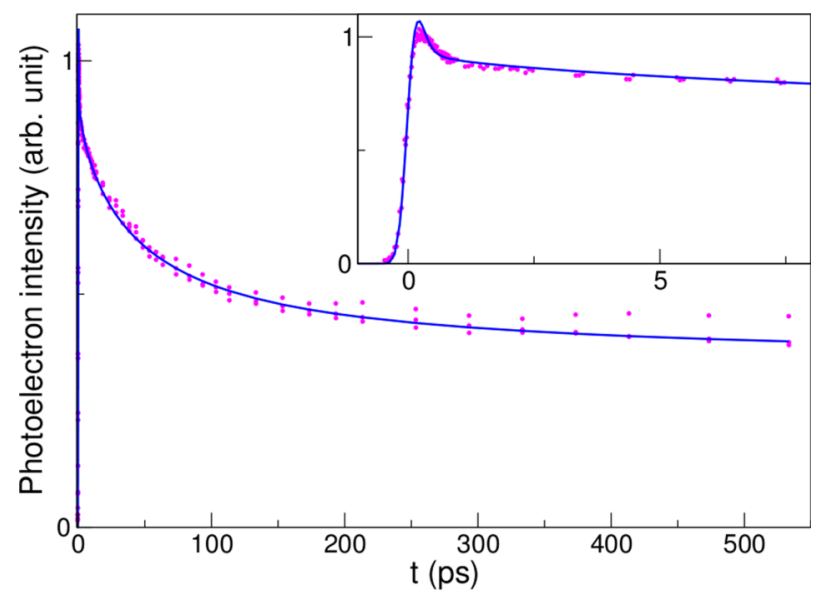

FIG. 5. The total photoelectron intensity as a function of time observed for $\mathrm{NaI}$ solution in methanol. The experimental data points are plotted with dots, and the solid line is the result of the least-squares fit to a diffusion model. 


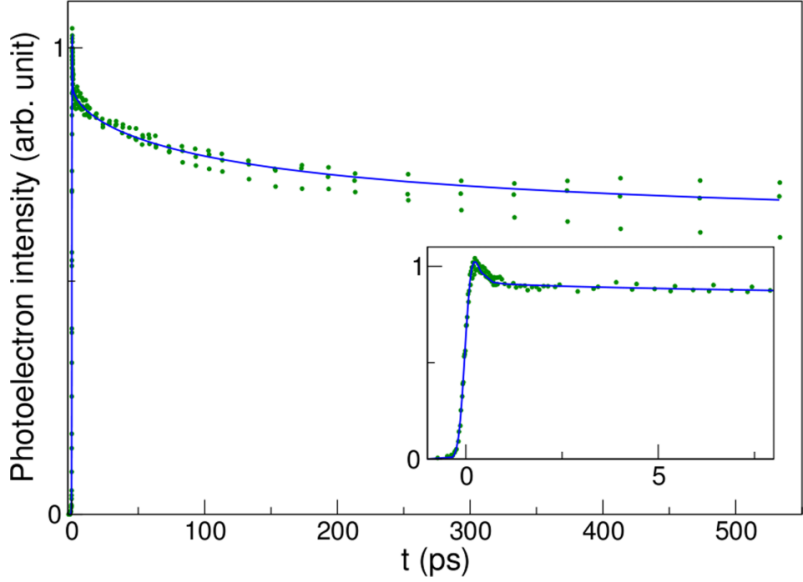

FIG. 6. The total photoelectron intensity as a function of time for NaI solution in ethanol. The experimental data points are plotted with dots, and the solid line is the result of the least-squares fit to a diffusion model.

attributed to the considerably larger diffusion coefficients that they assumed, which provides a deeper well for a given time profile. The mutual diffusion coefficients we employed for our simulation are listed in Table II and explained in the supplementary material. ${ }^{47}$

We found it possible to fit the observed time profile of the aqueous solution reasonably well without assuming any lifetime for $\mathrm{I}^{-*}$, if an interaction potential $V(r)$ is adjusted (Fig. 7). However, such fitting provides too large of a potential width $\beta^{-1}$ of about $0.3 \mathrm{~nm}$. On the other hand, the diffusion model analysis assuming a finite lifetime of $\mathrm{I}^{-*}$ (Table I) provides a $\beta^{-1}$ of $0.11 \mathrm{~nm}$ and a time constant $\tau_{a}$ of 0.38 ps. This result confirms that CTTS from $\mathrm{I}^{-}$(solv) occurs adiabatically with a finite $\mathrm{I}^{-*}$ lifetime.

\section{DISCUSSION}

\section{A. Ultrafast CTTS dynamics}

While the shortest time constant $\tau_{a}(0.25 \pm 0.01)$ determined for $\mathrm{D}_{2} \mathrm{O}$ agrees with our previous value $(0.31 \pm 0.1)$ within the experimental errors,${ }^{20}$ the new value is smaller than

TABLE II. Results obtained using a diffusion model.

\begin{tabular}{llllllll}
\hline \hline & $\mathrm{H}_{2} \mathrm{O}$ & $\mathrm{MeOH}$ & $\mathrm{EtOH}$ & $\mathrm{H}_{2} \mathrm{O}^{\mathrm{a}}$ & $\mathrm{H}_{2} \mathrm{O}^{\mathrm{b}}$ & $\mathrm{MeOH}^{\mathrm{c}}$ & $\mathrm{EtOH}^{\mathrm{c}}$ \\
\hline$T(\mathrm{~K})$ & $278^{\mathrm{d}}$ & $260^{\mathrm{d}}$ & $260^{\mathrm{d}}$ & 297 & 298 & 297 & 297 \\
$\Delta G / k_{\mathrm{B}} T$ & 2.4 & 1.4 & 0.92 & 3.0 & 4.1 & 3.6 & 3.1 \\
$\beta\left(\mathrm{nm}^{-1}\right)$ & 9.6 & 11 & 10 & 11 & 6.8 & $(10)^{\mathrm{e}}$ & $(10)^{\mathrm{e}}$ \\
$\mathrm{D}^{\prime} \times 10^{5}\left(\mathrm{~cm}^{2} / \mathrm{s}\right)$ & $3.0^{\mathrm{e}}$ & $1.6^{\mathrm{e}}$ & $0.78^{\mathrm{e}}$ & $8^{\mathrm{e}}$ & $5.8^{\mathrm{e}}$ & $13.1^{\mathrm{e}}$ & $6.6^{\mathrm{e}}$ \\
$\mathrm{r}_{0}(\mathrm{~nm})$ & $4.0^{\mathrm{e}}$ & $4.0^{\mathrm{e}}$ & $4.0^{\mathrm{e}}$ & $4.0^{\mathrm{e}}$ & 6.1 & $4.0^{\mathrm{e}}$ & $4.0^{\mathrm{e}}$ \\
$\tau_{a}(\mathrm{ps})$ & 0.38 & 0.13 & 0.13 & 0.2 & 0.22 & $\ldots$ & $\ldots$ \\
$\tau_{b}(\mathrm{ps})$ & $\ldots$ & $\ldots$ & $\ldots$ & $\ldots$ & 0.7 & $\ldots$ & $\ldots$ \\
$r_{a}$ & 0.70 & 0.52 & 0.57 & & & & \\
$\kappa_{r}(\mathrm{pm} / \mathrm{ps})$ & 8.1 & 2.3 & 0.55 & 5.2 & & & \\
\hline \hline
\end{tabular}

${ }^{\mathrm{a}}$ Reference 10 .

${ }^{\mathrm{b}}$ Reference 14 .

${ }^{\mathrm{c}}$ Reference 12 .

${ }^{\mathrm{d}}$ Estimated temperature.

${ }^{\mathrm{e}}$ Fixed values during fitting.

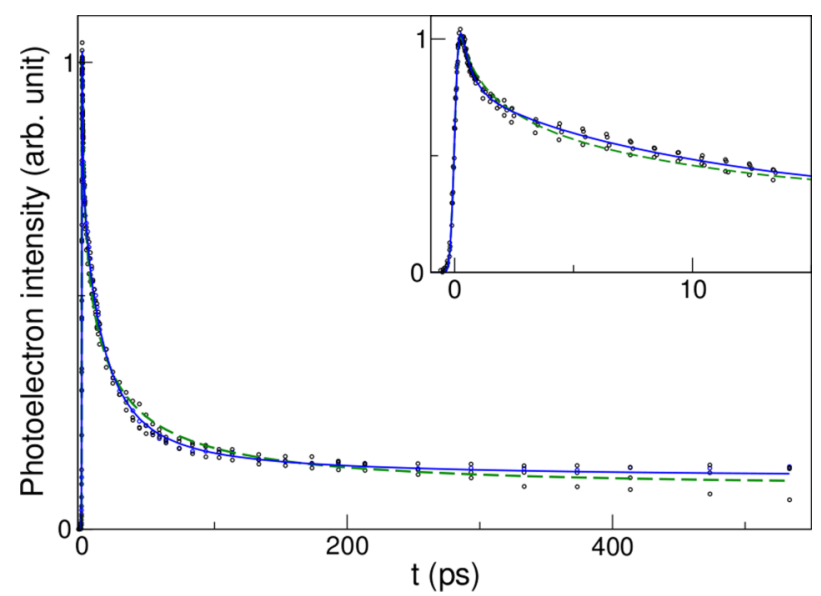

FIG. 7. The total photoelectron intensity as a function of time for aqueous $\mathrm{NaI}$ solution. The experimental data points are plotted with dots. The solid line is the result of the least-squares fit using a diffusion model with a metastable $\mathrm{I}^{-*}$, while the dashed line is the result obtained without assuming any metastable state. See the text for the assumed parameters.

the previous one. Consequently, the new $\tau_{a i}(i=1$ or 2 ) values for $\mathrm{H}_{2} \mathrm{O}$ and $\mathrm{D}_{2} \mathrm{O}$ become almost indistinguishable, which indicates that the CTTS dynamics in this time range have a negligible deuterium isotope effect and that the translation of water molecules plays an important role. It is possible that the librational response of water molecules plays a role at even shorter times $(<50 \mathrm{fs})$; however, this could not be confirmed in the present study due to our limited time resolution ( $0.3 \mathrm{ps})$. More importantly, the present study shows that the $\tau_{a 1}$ values in methanol and ethanol are considerably shorter than their longitudinal solvent relaxation times, indicating that the initial reaction step from $\mathrm{I}^{-*}$ to $\mathrm{CP}$ does not require significant reorientation of the solvent molecules.

As discussed earlier, estimation of the quantum yields of each species depends on the assumption of photodetachment cross sections of $\mathrm{I}^{-*}, \mathrm{CP}, \mathrm{SS}$, and $\mathrm{e}^{-}$(solv). The photodetachment cross section is essentially the same for $\mathrm{H}_{2} \mathrm{O}$ and $\mathrm{D}_{2} \mathrm{O}$ within the Born-Oppenheimer approximation; therefore, the different $r_{a}$ values extracted for $\mathrm{H}_{2} \mathrm{O}$ and $\mathrm{D}_{2} \mathrm{O}$ in our previous study ${ }^{20}$ suggested that internal conversion takes place from the $\mathrm{I}^{-*}$ state. However, in the present study, we found similarly high $r_{a}$ values $(0.9)$ for $\mathrm{H}_{2} \mathrm{O}$ and $\mathrm{D}_{2} \mathrm{O}$, which cannot be regarded as strong evidence for internal conversion from $\mathrm{I}^{-*}$ in water. On the other hand, the $r_{a}$ values in alcohols are lower than that in water. Based on the similarities in the UV absorption spectra of $\mathrm{I}^{-}$and the eBE of solvated electrons between water and alcohols, it is unlikely that the photodetachment cross sections differ significantly between water and alcohols. Therefore, we believe that internal conversion occurs from $\mathrm{I}^{-*}$ at least in alcohol, which can be examined by observing the ground state bleach recovery of $\mathrm{I}^{-}$.

\section{B. Recombination and diffusion dynamics at thermal equilibrium}

The mutual diffusion coefficients $\left(D^{\prime}\right)$ for a pair of $\mathrm{e}^{-}$(solv) and iodine under our experimental conditions are 
estimated to be $3.1,2.5,1.7$, and $0.8 \times 10^{-5} \mathrm{~cm}^{2} / \mathrm{s}$ for $\mathrm{H}_{2} \mathrm{O}$, $\mathrm{D}_{2} \mathrm{O}$, methanol, and ethanol, respectively. Vilchiz et al. have argued that their rate constant $k_{d}$, which corresponds to $\left(\tau_{c 1}\right)^{-1}$ in our kinetic model, is proportional to the estimated $D^{\prime}$ value. ${ }^{12}$ We found that $\left(\tau_{c 1}\right)^{-1}$ gradually decreases with $D^{\prime}$; however, we did not find a linear relationship between $\left(\tau_{c 1}\right)^{-1}$ and $D^{\prime}$. This difference between the two studies is attributed to the $D^{\prime}$ values estimated inaccurately by Vilchiz et al. based on the viscosities of the solvents. ${ }^{12}$ We note that the diffusion coefficient of $\mathrm{e}^{-}$(solv) does not scale with the viscosities of the solvents; for example, $D^{\prime}$ is larger in $\mathrm{H}_{2} \mathrm{O}$ than in methanol, even though the former is more viscous than the latter.

Using the diffusion model and estimated $D^{\prime}$ values listed above, we found that the depth $(\Delta G)$ of the attractive potential of mean force diminishes in the order of $\mathrm{H}_{2} \mathrm{O}>$ methanol $>$ ethanol. We have previously estimated that the chargeinduced dipole interaction between a solvated electron and neutral iodine atom is rather weak..$^{20}$ Thus, the main source of the potential of mean force is considered to be the hydrogenbonding network, and the observed well depth appears to scale with the number of hydrogen bonds created per unit volume. Considering the molecular size of alcohols, the value of $r_{\min }=0.4 \mathrm{~nm}$ assumed in the diffusion analysis may be too small. However, as we mentioned earlier, assumption of a larger $r_{\min }$ value provides a smaller well depth for alcohols, so the observed trend is unaltered by assuming longer $r_{\min }$ values for alcohols.

One of the clearest isotope effects observed in our previous study ${ }^{20}$ was for $\tau_{c 2}$, and we speculated that it originates in part from the zero point energy difference between liquid $\mathrm{H}_{2} \mathrm{O}$ and $\mathrm{D}_{2} \mathrm{O}$. However, the comparison with alcohols in the present study enables a more systematic consideration of this problem. Here, we find that the quantum yield of $\mathrm{e}^{-}$(solv) and the diffusion coefficient are anticorrelated: the slower the diffusion, the smaller the yield of recombination. This counter-intuitive result indicates that the recombination time constant $\tau_{c 2}$ differs greatly among the solvents. In fact, our kinetic model provides $\tau_{c 2}$ values of 30,150, and $450 \mathrm{ps}$ for water, methanol, and ethanol, respectively, in good agreement with the values of 29, 220, and 830 ps obtained by Vilchiz et al. ${ }^{12}$ The results are also consistent with the diffusion model, which yields respective $\kappa_{\mathrm{r}}$ values of 8.1, 2.3, and $0.55 \mathrm{pm} / \mathrm{ps}$ for water, methanol, and ethanol. Zusman has shown that an adiabatic electron transfer reaction rate is approximated by ${ }^{48}$

$$
k_{E T}=\frac{1}{\tau_{L}} \sqrt{\frac{\lambda}{16 \pi k_{B} T}} \exp \left[-\frac{\left(\Delta G^{0}+\lambda\right)^{2}}{4 \lambda k_{B} T}\right],
$$

where $\tau_{L}$ is the solvent longitudinal relaxation time given by $\left(\varepsilon_{\infty} / \varepsilon_{0}\right) \tau_{D}$, in which $\varepsilon_{\infty}$ and $\varepsilon_{0}$ are, respectively, the dielectric constants at high and low frequency limits, and $\tau_{D}$ is the dielectric relaxation time of the solvent. $\Delta G^{0}$ is the free energy of reaction, and $\lambda$ is the reorganization energy. The value of $\lambda$ in a dielectric continuum may be approximated as

$$
\lambda=(\Delta e)^{2}\left\{\frac{1}{2 a_{1}}+\frac{1}{2 a_{2}}-\frac{1}{R}\right\}\left\{\frac{1}{\varepsilon_{\infty}}-\frac{1}{\varepsilon_{0}}\right\},
$$

where $\Delta e, a_{1}, a_{2}$, and $R$ are the amount of transferred charge, the radii of the donor and acceptor, and the distance between their centers. The last term $\left\{\frac{1}{\varepsilon_{\infty}}-\frac{1}{\varepsilon_{0}}\right\}$ slightly diminishes from 0.55 for water to 0.50 for ethanol; however, this difference is small, because the electronic responses of these solvents are similarly fast. Soft X-ray photoemission spectroscopy shows that the eBEs of $\mathrm{I}^{-}$in water and methanol are 8.0 and $7.4 \mathrm{eV}$, respectively. In addition, the eBEs of $\mathrm{e}^{-}$(solv) in water and methanol are estimated to differ by less than $0.2 \mathrm{eV}$, although their absolute values may still contain ambiguities. ${ }^{28}$ Thus, although $\Delta G^{0}$ may be slightly larger for water than for alcohols, $\Delta G^{0}+\lambda$ is expected to be similar. If that is the case, $k_{E T}$ will scale crudely with $1 / \tau_{L}$. In fact, the ratio of $\tau_{c 2}$ values for $\mathrm{H}_{2} \mathrm{O}$, methanol, and ethanol, 30:150:450, is similar to the ratio of $\tau_{L}$ values, 0.2:3:10. Our diffusion model provides the same trend for the ratio of $1 / \kappa_{r}$ values, 0.12:0.43:1.8.

As we described in our previous study, ${ }^{20}$ the quantum yield of $\mathrm{e}^{-}$(solv) diminishes at lower temperatures (Fig. 7 of Ref. 20). It indicates that the temperature dependence of geminate recombination is weaker than that of diffusion; therefore, the activation energy for recombination is small. Thus, the pre-exponential factor of Equation (14), which is proportional to $1 / \tau_{L}$, becomes important for the recombination rate constants.

\section{CONCLUSION}

The charge-transfer-to-solvent reactions from $\mathrm{I}^{-}$to three polar protic solvents, water, methanol, and ethanol, were studied by time-resolved photoelectron spectroscopy of liquid microjets using a magnetic bottle time-of-flight spectrometer with variable pass energy. The results can be summarized as follows:

1. The elementary reaction step from $\mathrm{I}^{-*}$ to a contact pair occurs in less than 0.3 ps in water and 0.5 ps in alcohols. The sub-picosecond dynamics in $\mathrm{H}_{2} \mathrm{O}$ and $\mathrm{D}_{2} \mathrm{O}$ are quite similar, indicating that they are dominated by translational motion. The librational response of water was not detectable with our time resolution $(0.3 \mathrm{ps})$. The fact that $\mathrm{CP}$ is formed on the sub-picosecond time scale in ethanol indicates that it is formed without significant solvent reorganization in the long-range polarization field preexisting for $\mathrm{I}^{-}$. The quantum yield of a contact pair is $>0.9$ in water and $0.7-0.8$ in alcohols, which suggests that a faster solvent response time is essential to prevent internal conversion.

2. The overall quantum yield of solvated electrons is predominantly determined by geminate recombination/diffusion dynamics. The yield diminishes in the order of ethanol $>$ methanol $>$ water, because the geminate recombination rate increases in the order of ethanol $<$ methanol $<$ water. The relative recombination rates in the four solvents are correlated with the longitudinal relaxation times of the solvents, as predicted by transition state theory for an adiabatic outer-sphere electron transfer process. 
3. The diffusion model analysis indicates that the potential of mean force for an electron and iodine has a well depth of $2.4 k_{\mathrm{B}} T, 1.4 k_{\mathrm{B}} T$, and $0.92 k_{\mathrm{B}} T$ in water, methanol, and ethanol, respectively.

\section{ACKNOWLEDGMENTS}

This work was supported by JSPS KAKENHI Grant No. 15H05753. We thank Yo-Ichi Yamamoto for his experimental assistance and Huan Shen and Takura Horio for their contributions in the early stage of this work.

${ }^{1}$ E. Rabinowitch, Rev. Mod. Phys. 14, 0113 (1942).

${ }^{2}$ M. J. Blandamer and M. F. Fox, Chem. Rev. 70, 59 (1970).

${ }^{3}$ X. Y. Chen and S. E. Bradforth, Annu. Rev. Phys. Chem. 59, 203 (2008).

${ }^{4}$ W. S. Sheu and P. J. Rossky, J. Am. Chem. Soc. 115, 7729 (1993).

${ }^{5}$ W. S. Sheu and P. J. Rossky, J. Phys. Chem. 100, 1295 (1996).

${ }^{6}$ D. Borgis and A. Staib, J. Chem. Phys. 104, 4776 (1996).

${ }^{7}$ A. Staib and D. Borgis, J. Chem. Phys. 104, 9027 (1996).

${ }^{8}$ S. E. Bradforth and P. Jungwirth, J. Phys. Chem. A 106, 1286 (2002).

${ }^{9}$ A. Iwata et al., Chem. Phys. Lett. 207, 137 (1993).

${ }^{10}$ J. A. Kloepfer et al., J. Chem. Phys. 113, 6288 (2000).

${ }^{11}$ J. A. Kloepfer et al., J. Chem. Phys. 117, 766 (2002).

${ }^{12}$ V. H. Vilchiz et al., Radiat. Phys. Chem. 72, 159 (2005).

${ }^{13}$ H. Iglev, R. Laenen, and A. Laubereau, Chem. Phys. Lett. 389, 427 (2004).

${ }^{14}$ H. Iglev et al., Chem. Phys. Lett. 403, 198 (2005).

${ }^{15}$ M. K. Fischer, A. Laubereau, and H. Iglev, Phys. Chem. Chem. Phys. 11, 10939 (2009)

${ }^{16}$ C. G. Elles et al., J. Chem. Phys. 128, 061102 (2008).

${ }^{17}$ V. T. Pham et al., J. Am. Chem. Soc. 133, 12740 (2011).

${ }^{18}$ Y. Tang et al., Phys. Chem. Chem. Phys. 12, 3653 (2010).

${ }^{19}$ Y. Tang et al., Chem. Phys. Lett. 494, 111 (2010).
${ }^{20}$ Y. I. Suzuki et al., Chem. Sci. 2, 1094 (2011).

${ }^{21}$ F. Messina et al., Nat. Commun. 4, 2119 (2013).

${ }^{22}$ M. H. Elkins, H. L. Williams, and D. M. Neumark, J. Chem. Phys. 142, 234501 (2015).

${ }^{23}$ M. D. Sevilla et al., J. Phys. Chem. A 101, 2910 (1997).

${ }^{24}$ F. Uhlig, O. Marsalek, and P. Jungwirth, J. Phys. Chem. Lett. 3, 3071 (2012).

${ }^{25}$ F. Uhlig, O. Marsalek, and P. Jungwirth, J. Phys. Chem. Lett. 4, 338 (2013).

${ }^{26}$ F. Uhlig et al., J. Phys. Chem. A 118, 7507 (2014).

${ }^{27}$ Y. Yamamoto et al., Phys. Rev. Lett. 112, 187603 (2014).

${ }^{28}$ Y. Yamamoto et al., J. Phys. Chem. A 120, 1153 (2016).

${ }^{29}$ M. Faubel, S. Schlemmer, and J. P. Toennies, Z. Phys. D: At., Mol. Clusters 10, 269 (1988).

${ }^{30} \mathrm{M}$. Faubel and T. Kisters, Nature 339, 527 (1989).

${ }^{31}$ K. R. Wilson et al., Rev. Sci. Instrum. 75, 725 (2004).

${ }^{32}$ N. Kurahashi et al., J. Chem. Phys. 140, 174506 (2014)

${ }^{33}$ S. Thurmer et al., Phys. Rev. Lett. 111, 173005 (2013).

${ }^{34}$ Y. I. Suzuki et al., Phys. Rev. E 90, 010302 (2014).

${ }^{35}$ M. Michaud, A. Wen, and L. Sanche, Radiat. Res. 159, 3 (2003).

${ }^{36}$ T. L. Gilton, C. P. Dehnbostel, and J. P. Cowin, J. Chem. Phys. 91, 1937 (1989).

${ }^{37}$ R. N. Barnett, U. Landman, and A. Nitzan, J. Chem. Phys. 93, 8187 (1990).

${ }^{38}$ S. K. Jo and J. M. White, J. Chem. Phys. 94, 5761 (1991).

${ }^{39}$ F. Buchner, T. Schultz, and A. Lubcke, Phys. Chem. Chem. Phys. 14, 5837 (2012).

${ }^{40}$ R. L. Kurtz et al., J. Electron Spectrosc. Relat. Phenom. 40, 35 (1986).

${ }^{41}$ E. B. Krissinel and N. Agmon, J. Comput. Chem. 17, 1085 (1996).

${ }^{42}$ K. R. Siefermann et al., Nat. Chem. 2, 274 (2010).

${ }^{43}$ A. Lubcke et al., Phys. Chem. Chem. Phys. 12, 14629 (2010).

${ }^{44}$ A. T. Shreve, T. A. Yen, and D. M. Neumark, Chem. Phys. Lett. 493, 216 (2010).

${ }^{45}$ H. A. Shen et al., Chem. Lett. 39, 668 (2010).

${ }^{46}$ A. T. Shreve, M. H. Elkins, and D. M. Neumark, Chem. Sci. 4, 1633 (2013).

${ }^{47}$ See supplementary material at http://dx.doi.org/10.1063/1.4960385 for the diffusion coefficients employed in our analysis.

${ }^{48}$ L. D. Zusman, Chem. Phys. 80, 29 (1983). 